\title{
Evidence of Fab fragment gene in an invertebrate: The sea star Asterias rubens
}

\author{
Michel Leclerc ${ }^{*}$ and Nicolas Kresdorn ${ }^{2}$ \\ ${ }^{1} 556$ rue Isabelle Romée, 45640 Sandillon, France \\ ${ }^{2}$ GenXpro de, Frankfurt, Germany
}

\begin{abstract}
Fc receptor gene, was just discovered with the emergence of Fab fragments gene, in Asterias rubens. These last ones were described in Ig light chain and Ig heavy chain, when compared to mouse genome.
\end{abstract}

\section{Introduction}

The purpose of this article is to draw attention to the mass of information that has accumulated on the primitive sea star antibody since about 1983. This work has emanated from our laboratory, those of Gothenburg (Dr Sam Dupont), those of GenX pro (Frankfurt), those of Fasteris (Switzerland).

Our purpose will be to recall, the homologies between the primitive antibody and the mouse Igkappa chain [1], the presence of two Immunoglobulin sites in this last one, the specificity of this " antibody " to the the antigen HRP which induces the reaction: " antigen-antibody ", at last the existence in the sea star immune system of the Fc receptor gene [2].

We present now, the last results, concerning this primitive antibody : the emergence of Fab fragments in the immunized and nonimmunized sea star transcriptomes, when compared to mouse genome.

\section{Materials and methods}

Sea stars were obtained from the Biology Institute (Gothenburg University)

Immunizations to HRP (Horse-radish peroxydase), genomic studies were already described [1].

Briefly, after ligation of adapters for Illumina's GSII sequencing system, the cDNA was sequenced on the Illumina GSII platform sequencing.

$1.100 \mathrm{bp}$ from one side of the approximately $200 \mathrm{bp}$ fragments sequences were assembled using Velvet [3].

\section{Results}

First a comparison between non-immunized sea star genome and mouse genome was performed.

Studies were compared to mouse Immunoglobulin Light chain, then to heavy chain:

One contig (Contig9579) could be annotated via TBLASTX to Mus musculus "Mus musculus immunoglobulin IgG2a light chain Fab fragment (Mab10CK) mRNA, partial cds" from the NCBI database (gi|2852986|gb|AF042656.1|), with an e-value of 4.15e-05. On an aligned region of 21 amino acids, 14 positive and 9 identical amino acids were found.

T C A C C A G G T G C T G T G T T G A C A T C A G C A C T T T C T GA A T C C A C C T C C A T T G G T T G A T C C A C T T C C T C T T T C A C T A TA G T TA C G A T T T G A T A T C A A C A C T G T C A C C T G T A A G C G C A T C A A C A C C G C T T G T T A T A T C A A C A G A C GATCTCCTTTGTGGATCCACTTTTTGATCGGTGG A T A G A G G C G GA G T G G A T G A C T C C A T C T T T G C T A A T G T T T T G T C A T C G G C T A A T G T T GA T G A A C T T T C T T C A G C T T T T A C C T C G G T C T T C A C A T C C A C T G T T T G G T T T T C T G A T T T C A C G G A A A C A G T T G A T G T C G T T T T GA C T G G A T C C A C T GA C T T A T C A G C C A A T G T T C C C T G C G G A T T G T T GA T G T A C T T T G T T GA GA GA T G G C T G T G A A G G G A T T G T G G C G G G A T C G A A G G A A G C T C C T A A G C C T T C C T C C C C T G G G G T G G C G T T T C T A GA A G C G A G GA A T C T T T G G C A T C T T C GA A A C A C A A GGGA T GGTA T G TC C T GGA G T T G A A C C T G A C A G C A T C T C T C A G T T T C C A A A C C T C T T CA T C T T C A T C A A A GAAT T GC T C A A A C G G C T C A T G A C A C A G A G A A C A C A T C T C A T C G C C A G T G A C G T T T C C A T C A A T A G C C A C A C A GA G G A T G C C A T C T T T A T CA G G T T A G A C T C T G C A G T C A T C T C C T C A A A G A A A C T GCTCCTCTCT TCA TCCACTTCGTTGATTTCTTCAA A G T C C A A C C A C T G A T C GA C T T C A T A GA A C C A C T T C C T G G A C T G A A C A G C C T T C A C T C C A T

Correspondence to: Michel Leclerc, 556 rue Isabelle Romée, 45640 Sandillon, France

Received: August 19, 2016; Accepted: September 13, 2016; Published: September 16, 2016 
C C T T C T C A C G A C G A T T C T G A C G G A A G T G C C A G T C C A G A T G T T T C C G A T A T C T G T C C A T T T CATCAATGACAAAGCGCATACCA3'

Second, results with mouse Ig heavy chain were obtained, when compared to mouse genome:

One contig (c37102_g1_i1) could be annotated via TBLASTX to Mus musculus "Mus musculus immunoglobulin IgG2a heavy chain Fab fragment (Mab10CH) mRNA, partial cds" from the NCBI database (gi|2852988|gb|AF042657.1|), with an e-value of 6.1e-06. On an aligned region of 20 amino acids, 14 positive and 10 identical amino acids were found.

5' A A C T G C G C C A C C A G C A A G T A T G C C G TACCCTTCGGCTATTGCGACGGCACCAGACTACCC C A A A G C A A T G G C A G C A T C G G C C C C T C C A T T T G G T G G T G A A G C T C C G C C C C C A A G T T A C G A G G A G G C T G T T G G C A A A A C A G A C A G A C T G G C C A A C G C A A G G A A C G A T G A G G A T T A C G T C A T T G A C G A T C A G T T C G T G C C T A G T T A T C C G T A C T T C A A C C T T T C G G T T G A T G T A C C A C C G G G TTCCCGCCATTAACCCCATGGAGTTTAAAGAGA3'

At last, results with immunized sea star genome were given when compared to mouse IgG2a light chain :

One contig (Contig5278|m.6828) could be annotated via TBLASTX to Mus musculus "Mus musculus immunoglobulin IgG2a light chain Fab fragment (Mab10CK) mRNA, partial cds" from the NCBI database (gi|2852986|gb|AF042656.1|), with an e-value of 1.62e-05. On an aligned region of 38 amino acids, 23 positive and 14 identical amino acids were found.

5'TCTTTGGA A A C C C CGA A C CGGAACTGA C C TGG C A G A G G A A G A G C C C A G C A G G T G C A G A C C T A C C A C T C G G T G C A G A G C T C C G A T T C A A C A A C C A G C A T T T G A T C C T C A G G A A T A T A C A G A T T G A A G A T G C T G G G G A G T A T G A A T G T A T G A C C A C T A A T G T T G A G GA A G A C A T C T G G A A C C A T T A C T G T C C G A G T C C C A C C C A C C T G G C T G C A A G A A T C T C T G A C A T C GA G G G A A C A T C T A C G G A G A C T T A T C C A T G A A G T G T A A A G C A GA GA G T C C C G A T G G CGT A A C C T A C A C A T G G T A C C G C A A T G C T G A G G T G A T C CAAGACCTTCCCAGGCATCAGTTCAGCAACGGTTTGA A GA C A C T C A C C A T C C A G A A T C T G A C T G T G G A A G A C A C C G G G A T G T A C C A G T G T A T G G C T A
G T A A T G A A T A C G G C C A G A T A T A C A C C A C A G C A C A G G T C A C T G T A C A A G C C A T T G C A C C A T G G T T T G A G A C G C C C C T T G A G G T G G A C C A G CC A G C A C C C C G T G G T G G C T C A G T G A C C A T C C T C T G C C A A C C T A A A G C T G C A C C C A C A C C C G T C A T C G T C T G G A C C A A A G G A A G C A G A C C A T T G A G A A C G G T G G C C G C T A C A C C G T C A T G GA C A A C G G T G A T T T G A T G A T C A G T G G T G T T A GT GA T G CA GA T G GA G GA GA C T A C A C C T G T A C G G C T A C C A A T A G C A T G G G A A C T G A T T C A A G C A C T G G T A G C T T GA T C A T C A A G GA T G G T A C A G C C A T G G T G G A G C C A C C C A T G G C A T T G A C T G T A A A C G A A G G G A G A T G G C A C A T T GA C C T GC C G T GCA T C A C A C GA T C C G T T C T T T G A GCTGGA GT A T G T G T G GC T GA A A GA A G GA A T C C A A G T T G A T C C T C G T C T C C C T A A C T A T G A GA TACCTCAGA TCCAAATGGGCGAGCCAAGTC A G C T G C A T A T C A A A G A C G C C A C T A T G G ATCTAGGAGGGGAGTACATGTGC3'

\section{Discussion and conclusion}

It is clear that mouse Fab IgG2a light chain gene, mouse Fab IgG2a heavy chain gene, are present in the sea star genome. It is also noticeable that these genes, show a "symmetry ", or at least a similarity in their configurations: we count on an aligned region of 21 amino-acids, 14 positive and 9 identical in the case of IgG2a light chain, 14 positive and 10 identical in the case of IgG2a heavy chain out of 20 amino-acids. As for immunized sea star IgG2a light chain which is only studied, in the present work : the number of positive amino-acids increases, in a significant manner (on an aligned region of 38 amino-acids, 23 positive instead 9 in non-immunized sea stars to HRP : undoubtly it is due to immunization (further studies are bound to unravel the phenomenon).

In addition to the work on Fc receptor gene [2], it appears that Fab fragments gene, corroborate the existence of the Invertebrate Primitive Antibody through the Asterias rubens history [4].

\footnotetext{
References

1. Vincent N, Osteras M, Otten P, Leclerc M (2014) A new gene in A. rubens: A sea star Ig kappa gene. Meta Gene 2: 320-322. [Crossref]

2. Leclerc M (2016) Immunol Lett submitted

3. Zerbino DR (2007) Asterias rubens: Evidence of NF-Kappa B Genes. Gen Res 18: 821-829.

4. Leclerc M (2016) Sea star Genomic Studies Following 2.
}

Copyright: (C2016 Leclerc M. This is an open-access article distributed under the terms of the Creative Commons Attribution License, which permits unrestricted use, distribution, and reproduction in any medium, provided the original author and source are credited. 